Neurosurg Focus 10 (5):Introduction, 2001, Click here to return to Table of Contents

\title{
ORBITAL TUMORS
}

\author{
Neurosurgical Focus, May 2001 \\ Volume 10, Issue 5
}

\author{
Topic Editors: Aaron S. Dumont, M.D., John A. Jane, SR., M.D., Ph.D., F.R.C.S.(C) \\ Department of Neurological Surgery, University of Virginia, Charlottesville, Virginia
}

Lesions afflicting the orbit have been apparent since antiquity. A comprehensive understanding of the nature and treatment of such conditions, however, has not been realized until more recently. Indeed, initial approaches to the surgical management of orbital tumors were met with considerable trepidation. The evolution in the surgical management of orbital lesions has been facilitated by the multidisciplinary involvement of specialists from neurosurgical, ophthalmological and craniomaxillofacial disciplines. The present issue of Neurosurgical Focus is devoted to orbital tumors and includes six articles encompassing a variety of topics pertinent to a contemporary perspective on orbital lesions and their management.

In the first article, Darsaut and colleagues provide an overview of orbital tumors. They present a classification scheme for orbital tumors and discuss orbital tumors from a pathoanatomical standpoint. Clinical presentation, radiological findings, and principles of treatment are also developed. The article serves as a useful introduction to those who are less familiar with orbital lesions and also provides a succinct review for the experienced orbital surgeon.

In the next two articles, experienced neurological and ophthalmological surgeons address approaches to the orbit. In the first article Bejjani and coworkers detail extraorbital approaches, and in the second Cockerham and colleagues outline transorbital approaches to orbital tumors. Bejjani and coworkers first provide an overview of surgical approaches to the orbit. The rationale behind choosing the appropriate surgical approach for a given patient is then discussed. They describe the inferior orbital approach or posteroinferior orbitotomy, frontotemporal approach to superior orbital fissure tumors, frontoorbitotemporal approach to optic nerve tumors, and frontotemporal (pterional approach) for sphenoid wing meningiomas. Surgical adjuvants including neuronavigation, endoscopy, and radiotherapy are also addressed. The use of frameless stereotaxy (neuronavigation) is further discussed in the fifth article by Boulos and coworkers. Cockerham and colleagues address transorbital approaches for orbital tumors. Although traditionally heralded as superfluous to the neurosurgeon, a rudimentary knowledge of such approaches is important. Critical anatomical points and important tenets of orbital surgery are introduced that are helpful to any surgeon treating patients harboring orbital lesions. Techniques of anterior orbitotomy, lateral orbitotomy, transconjunctival medial orbitotomy, and mediolateral orbitotomy, with their associated merits and limitations, are presented.

In the fourth article, DeMonte, et al., discuss complications of surgery of lesions traversing or primarily involving the orbit. They retrospectively analyze their experience with 56 patients who underwent 57 anterior or anterolateral skull base tumor resections during which partial orbital bone excision was performed. They assert that extensive reconstruction is not necessary in the majority of cases; however, primary reconstruction is mandated in cases of orbital floor defects. Their careful analysis provides scientific validity to many of the commonly held beliefs and practices of those well versed in surgery in and around the orbit.

Boulos and coworkers discuss the topic of meningiomas involving the orbit primarily or secondarily. A historical perspective is provided as is discussion of the presentation and clinical findings, imaging characteristics, pathological features, and methods of primary and adjuvant therapy. A case illustration is complete with intraoperative video clips. Although some have found frameless stereotaxy unhelpful in the management of orbital tumors, these authors are proponents of its use, particularly in cases requiring the resection of the intraosseous portions of meningiomas that so commonly occur with orbital involvement.

In the sixth article the authors address fibrous dysplasia. A comprehensive overview is provided and the authors draw on their own experience in managing this entity. Emphasis is placed on the origins of visual impairment and the surgical management from both cosmetic and functional visual perspectives. The authors introduce the notion that cystic degeneration, particularly with involvement of the anterior clinoid process, is the most common cause of visual loss in patients with fibrous dysplasia. They advocate against routine optic nerve decompression for treatment.

Since the pioneering efforts of early surgeons targeting lesions of the orbit, considerable advancements in our understanding and treatment of orbital tumors have occurred. Although traditionally fraught with complications, surgical approaches primarily or secondarily involving the orbit have become commonplace for neurosurgeons and their colleagues. Orbital tumors can now be treated with efficacy and safety by experienced and often multidisciplinary surgical teams. With further developments in understanding of orbital tumors, refinements in surgical technique, delineation of the utility of surgical adjuncts (including intraoperative imaging), adjuvants therapy, and of course careful patient follow up, improvements in patient outcome will hopefully emerge.

The editors would like to extend their gratitude to the all of the reviewers including Mark Berenstein, M.D., Christopher Bogaev, M.D., Johnny B. Deleshaw, Jr., M.D., J. Max Findlay, M.D., Ph.D., Gregory A. Helm, M.D., Ph.D., Edward R. Laws Jr., M.D., John A. Persing, M.D., James T. Rutka, M.D., and Garnette R. Sutherland, M.D. The editors would also like to thank Susan Robinson and the entire staff of the Neurosurgical Focus office for their patience, efficiency, and support. The editors also thank contributing authors for their commendable work. 